\title{
Memory bias for social hierarchical information is modulated by perceived social rank
}

\section{Kirsty Griffiths, Jason Stretton \& Tim Dalgleish}

To cite this article: Kirsty Griffiths, Jason Stretton \& Tim Dalgleish (2022): Memory bias for social hierarchical information is modulated by perceived social rank, Memory, DOI: 10.1080/09658211.2022.2029902

To link to this article: https://doi.org/10.1080/09658211.2022.2029902

\section{(c) 2022 The Author(s). Published by Informa UK Limited, trading as Taylor \& Francis} Group

曲 Published online: 07 Feb 2022.

Submit your article to this journal $\widetilde{ }$

Џ Article views: 98

Q View related articles

View Crossmark data $\nearrow$ 


\title{
Memory bias for social hierarchical information is modulated by perceived social rank
}

\author{
Kirsty Griffiths (10 a, Jason Stretton ${ }^{\mathrm{a}}$ and Tim Dalgleish ${ }^{\mathrm{a}, \mathrm{b}}$ \\ ${ }^{\mathrm{a}}$ Medical Research Council Cognition and Brain Sciences Unit, University of Cambridge, Cambridge, UK; ${ }^{\mathrm{b}}$ Cambridgeshire and \\ Peterborough NHS Foundation Trust, Cambridge, UK
}

\begin{abstract}
Hierarchies pervade human society, characterising its members along diverse dimensions ranging from their abilities or skills in a particular domain to their economic status or physical stature. One intriguing aspect of the centrality of hierarchies, relative to egalitarian constructs, is that hierarchically-organised social information appears to be remembered more easily than non-hierarchically-organised information. However, it is not yet clear how one's social rank within a hierarchy influences processing. In a pre-registered study with 66 healthy participants, we examined memory recall for hierarchical information when participants themselves were positioned higher in the hierarchy versus lower in the hierarchy, both relative to an egalitarian control condition. The results replicate previous work showing that hierarchical information is memorised faster relative to the egalitarian control. Importantly, this effect was modulated by the participant's position within the hierarchy, with higher positioned participants memorising information faster than lowerpositioned participants. This study provides new evidence showing biases in memory will favour hierarchical information if modulated by perceptions of one's own social rank.
\end{abstract}

ARTICLE HISTORY

Received 17 June 2021

Accepted 11 January 2022

\section{KEYWORDS}

Memory; self-enhancement bias; social hierarchy; social rank

\section{Introduction}

Hierarchies pervade human society, characterising its members along diverse dimensions ranging from their abilities or skills in a particular domain to their economic status or physical stature (Zink et al., 2008). People typically engage in a wide range of goal-directed activities to maintain or enhance their position within the hierarchies that contextualise their lives, and current standing appears to be vigilantly monitored in the face of dynamic shifts in relative social rank (Anderson et al., 2015). Rank-relevant cues can be explicit (e.g., uniforms, rank-specific grammar, height, age) or implicit (e.g., non-verbal cues of dominance or submissiveness) with respect to valued social dimensions (Magee \& Galinsky, 2008). In sum, despite at least some cultural ideologies endorsing an egalitarian social structure, hierarchies remain the dominant system framing human social interaction (Bellah et al., 1996; Zitek \& Tiedens, 2012).

One intriguing aspect of the centrality of hierarchies, relative to egalitarian constructs, is that hierarchicallyorganised social information appears to be remembered more easily than comparable non-hierarchically-organised information. In one of a series of elegant studies, Zitek and Tiedens (2012; Experiment 2) presented participants with the names of strangers in either organised within prototypical social hierarchies or egalitarian arrangements, and asked them to remember the diagrams. Participants then attempted to recall these "organograms", and the number of exposures of the organogram required for successful recall was the variable of interest. Zitek and Tiedens (2012) showed that the hierarchy diagram was learned with the fewest exposures relative to the non-hierarchy diagrams. One of the non-hierarchy diagrams involved partitioning the participants into sub-groups to facilitate chunking in memory, thus mimicking the partitioning enshrined in hierarchical structures. The enhanced memorability for the hierarchy diagram was preserved even relative to the partitioned control diagram. In related experiments, Zitek and Tiedens (2012) showed that "fluency" for hierarchical information extended beyond the memory domain to encompass identification, decision making and liking.

Despite this literature showing enhanced fluency of hierarchical information, it is unclear how people's rank within a given hierarchy impacts processing. For example, does a manager who holds a higher rank in the hierarchy find it easier to remember information pertaining to their group's organisation over an assistant who holds a lower rank position? Although there is a dearth

CONTACT Kirsty Griffiths @ Kirsty.griffiths@mrc-cbu.cam.ac.uk

(4) Supplemental data for this article can be accessed https://doi.org/10.1080/09658211.2022.2029902

○ 2022 The Author(s). Published by Informa UK Limited, trading as Taylor \& Francis Group

This is an Open Access article distributed under the terms of the Creative Commons Attribution License (http://creativecommons.org/licenses/by/4.0/), which permits unrestricted use, distribution, and reproduction in any medium, provided the original work is properly cited. 
of relevant behavioural data, neuroimaging has shown individuals primed with high rank (relative to individuals primed with low rank) recruited greater activation of the dorsolateral prefrontal cortex, which is associated with perceptual-attention, saliency and other cognitive systems (Zink et al., 2008). This finding suggests that variations in an individual's rank could influence both attention and encoding when engaging in rank-based cognition.

How might an individual's social rank within a given hierarchy influence their memory for information pertaining to that hierarchy? In line with the literature on selfenhancement bias across multiple domains of cognition (Taylor \& Brown, 1988) and personal agency, our prediction was the participants of high ranking positions within hierarchies would show superior recall for the organisation of information than individuals positioned in low ranking positions or in non-hierarchical organograms. There is a wealth of evidence that mentally healthy individuals have positively enhanced, rather than realistic, self-perceptions across multiple cognitive domains. For example, identifying positive traits are far more characteristic of the self than negative attributes (Alicke, 1985; Brown, 1986). This is exemplified in the "Better-than-AverageEffect" where mentally healthy individuals tend to endorse positive traits as more self-descriptive relative to others (e.g., Alicke et al., 1995), and from data showing that self-evaluations are systematically more positive than those of objective observers (e.g.,Lewinsohn et al., 1980). This self-enhancement bias extends to attributions (see Mezulis et al., 2004), evaluations of the future (see Sharot, 2011) and, pertinent to the present study, to selfreferent memories. Mentally healthy individuals show better recollect for information relating to personal success than to personal failure (Silverman, 1964), are more likely to remember their own performance on tasks as more positive than it objectively was (Anderson et al., 2012; Crary, 1966; Gramzow et al., 2003), are quicker to retrieve autobiographical memories of positive compared to negative events (see Blaney, 1986; Rubin \& Berntsen, 2003), and report slower fading of positive affect to personal events, relative to negative affect, over time (e.g., Hitchcock et al., 2017).

Relatedly, high rank within a hierarchy tends to be positively regarded by both self and others as it brings a sense of personal agency over resources and other people (Magee \& Galinsky, 2008; Zitek \& Phillips, 2020). Those positioned high rank (as well as those with high power) are also stereotypically characterised as experiencing elevated greater positive affect, enhanced attention to rewards relative to threats, and less inhibited social behaviour (Cho \& Keltner, 2020; Keltner et al., 2003; Smith \& Trope, 2006; Tarr \& Kohles, 2019; Trope \& Liberman, 2010). Considering how a large part of our self-identity is made up of how we define ourselves in relation to others (Brewer \& Gardner, 1996), healthy individuals may show increased motivation to the task-relevant goal if the self is implicitly cued in a favourable position over others. Therefore, by placing an individual in a high-rank position over others within the hierarchy, we expect the self-enhancement bias will influence their attention in a top-down processing manner.

To test our proposal that elevated personal rank would bias memory for hierarchical social information, we adapted the memory paradigm from Zitek and Tiedens (2012). Participant's rank was manipulated by positioning the participant at the bottom or top of the hierarchy for the hierarchical organograms, and randomly in the egalitarian control organogram, in a between-groups design.

Our specific hypotheses were:

(1) Replicating the findings of Zitek and Tiedens (2012), that hierarchical organograms, regardless of participants' rank position, would be memorised more quickly (i.e., need fewer learning attempts) than the egalitarian organogram.

(2) For the hierarchical organograms, participants placed in the high-rank position would memorise the structure faster than individuals in the lowrank position.

\section{Methods}

The Cambridge University Psychology Research Ethics committee approved the study (PRE. 2017.083). We preregistered all measures, manipulations, samples sizes, and exclusion criteria, with further information available online (https://osf.io/b3yhw/).

\section{Participants}

Sample size was calculated based on effect size estimates from pilot data, with 21 participants required in each of the three organogram groups for a two-tailed alpha of .05 , and $80 \%$ power to detect an effect size of Cohen's $f=0.4$ for a main effect of condition. An additional five participants for each group were recruited to allow for any protocol failures, giving a total of 78 participants (aged 18-65) for the study. The study was advertised using the online participant panel at the MRC Cognition and Brain Sciences Unit, University of Cambridge. Participants were offered the opportunity to take part with three exceptions: (i) any history of neurological impairments, (ii) a self-declared learning disability; and (iii) any self-reported history of a mood disorder. In addition, participants who scored more than three standard deviations below the sample mean on the Rey-Osterrieth complex figure test (ROCF; see below) were set aside from analysis. In total, data for 66 participants (aged 18-64, 44 females) were available for full analysis, with data from 12 participants set aside. Participants were removed from the main analysis because either they withdrew from 
the study ( $n=3)$, the ROCF was below the sample mean ( $n=7 ; M=29.25, S D=3.43$ ), or they could not complete the organogram task $(n=2)$. Participants were compensated $£ 6$ for one hour of their time.

\section{Measures and materials}

\section{The organogram task}

The Organogram Task (Figure 1) assesses the participant's ability to memorise information about social structures. The task contains three conditions administered in a between- participants design, with participants allocated randomly to each condition. Participants are shown either an egalitarian organogram (Figure 1, Panel A), or a hierarchical organogram with the participant situated in a low rank position (LR hierarchy; Figure 1, Panel B), or in a high rank position (HR hierarchy; Figure 1, Panel B). The organograms were adapted from Zitek and Tiedens (2012). Each organogram comprises two components: the nodes (i.e., the names and faces of people) and associations (i.e., the relationships between people). Each organogram has 8 nodes and 12 associations to ensure comparability of cognitive load for memory recall across the three conditions. Although participants were only required to recall the names when remembering the organograms, the faces were included to reinforce the social nature of the study (Crivelli \& Fridlund, 2018). Implicit cues relating to social rank (e.g., age, gender, facial expression) were controlled by allocating participants to one of three stimuli-congruent groups based on their age (18-30; 31-45; 46-65 years) and gender. All facial expressions were emotionally neutral with eyes facing forward. The participant's own face and name were included within the organogram to manipulate their hierarchical position. A photo of the head and shoulders was taken at approximately $1 \mathrm{~m}$ distance using a digital camera. Organograms were presented via a computer screen coded using Matlab 2018a prior to the COVID-19 pandemic. Due to the pandemic-related social lockdown in the UK, the study transitioned to online testing, with the task presented on Microsoft Office PowerPoint v2016. For virtual testing the photo was taken via the videoconference application. After testing, the photo of the participant was deleted.

A pilot study with a separate sample of participants ( $n=30 ; 10$ in each condition) was carried out to validate how well the egalitarian and hierarchical structures represented their respective constructs and to derive an estimate of effect size (see Supplementary Materials).

In line with Zitek and Tiedens (2012), when presenting the task the experimenter explained to participants that they will be shown an organogram representing relationships within a group of people and that the task is to memorise the organogram structure and the names of the organogram members as quickly as possible. The experimenter did not mention the organogram was in any way linked to hierarchical social information. The organogram was then presented on the computer screen for $7 \mathrm{~s}$ (see Figure 1). Once the organogram disappeared from the screen, participants had to recall the organogram as best they could, using the pen and paper provided. There were no time constraints on how long it took the participant to recall the organogram, but participants were told to inform the experimenter when they had finished with their attempt. The experimenter then checked the organogram to assess the extent to which the nodes and associations were recalled correctly and in the correct positions. If the participant failed to recall the organogram completely accurately, the computer was reset to display the organogram again. This process was repeated until participants accurately duplicated the organogram. The number of times the organogram was (re-)presented constituted the dependent variable for analysis.

\section{The Rey-Osterrieth complex figure test (ROCF; Osterrieth, 1944)}

The ROCF is a neuropsychological assessment that is commonly used to evaluate an individual's visuospatial abilities, memory, attention, planning and working memory. Participants are shown a diagram of a complex structure and are asked to memorise it for $3 \mathrm{~min}$, after which the diagram is removed. Participants are then asked to recall the diagram from memory with the pen and paper provided, with no time constraints. In the current study, this process was completed once and took approximately $5 \mathrm{~min}$. Scoring involves dividing the figure into eighteen identifiable areas, each of which is marked in terms of the accuracy of its recollected position and any memory distortion exhibited. A maximum score of 36 is possible. The ROCF was used in the present study as a measure of general visuo-spatial recall ability to ensure comparability in this underlying skill across groups, as it is likely to be associated with overall task performance. Participants scoring more than three standard deviations below the sample mean on the ROCF were also set aside from analyses as they were deemed to have outlier visuo-spatial skills which would affect their ability to complete the Organogram Task.

\section{National adult reading test (NART; Nelson \& Willison, 1991)}

The NART provides a means of estimating verbal intelligence. The NART comprises 50 words printed in order of increasing obscurity, which participants must read aloud to the experimenter. The words are all irregular with respect to the common rules of pronunciation in order to minimise the possibility of reading by phonemic decoding rather than word recognition. The NART error score (i.e., total number of errors made on the complete NART) can be computed into an estimate of verbal intelligence which is age-standardised (see Nelson \& Willison, 1991). The NART was used here to allow evaluation of 


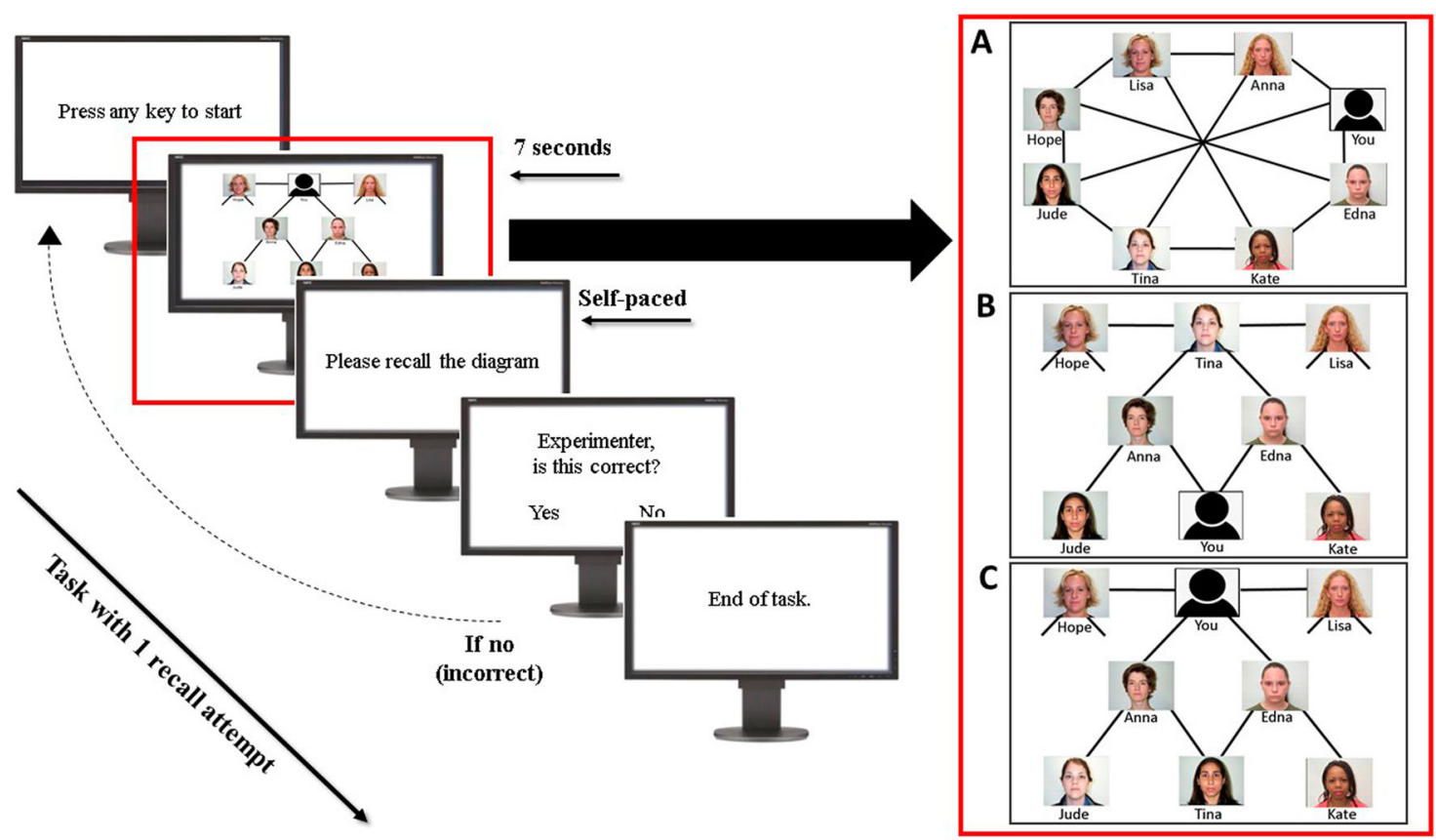

Figure 1. The Organogram Task.

comparability in estimated verbal intelligence across groups, as verbal intelligence is likely to be associated with overall task performance.

\section{Questionnaires}

The Beck Depression Inventory (BDI), the Social Comparison Scale (SCS), Social Dominance Orientation Scale (SDO) were included to allow assessment of group comparability on how participants characterise themselves in terms of social behaviour and mood. For more information on each questionnaire, see the supporting materials.

\section{Procedure}

Data collection was a mixture of in-person testing prior to the COVID-19 pandemic and online following the pandemic. Participants were randomly assigned to one of the three organogram conditions. A headshot photo of each participant was then taken (either via a digital camera or over a video-conferencing application) which was inserted into the relevant organogram (Figure 1). Participants then completed the NART and ROCF, followed by the Organogram Task and the questionnaires ${ }^{1}$, either in person or via a video-conference link. Participants were then debriefed, thanked, and compensated for their time. The process took approximately $1 \mathrm{~h}$ with testing and questionnaires, although for online testing participants completed the questionnaires at their own pace after the video conference ended.
Statistical analyses were conducted in SPSS version 27. Questionnaire measures with $>20 \%$ of individual item responses missing were treated as a missing value, and measures with $\leq 20 \%$ missing items imputed via multiple imputation.

\section{Results}

\section{Participant characteristics}

The final analysis sample consisted of 66 participants across the three organogram conditions: Egalitarian ( $n=$ 21); Low rank (LR) hierarchy $(n=23)$; and High rank (HR) hierarchy $(n=22)$. Table 1 presents the demographic, cognitive, mood and social data for the organogram groups.

The organogram groups appeared comparable in terms age, gender, NART error scores, educational history, employment status, testing location, mood and social cognition. Therefore, no covariates were included in the main analyses.

\section{Organogram Task performance}

To test our first hypothesis, data from the hierarchical organogram conditions (HR hierarchy and LR hierarchy) were combined to form one single "Pooled hierarchy" organogram group. Recall performance (See Figure 2) between the Pooled hierarchy group $(n=45)$ was compared to the Egalitarian group $(n=21)$. Following preregistration, a violation of normality was detected in the Pooled hierarchy group (Shapiro-Wilks $W=.93, p=.01$ ), therefore a non-parametric Mann Whitney $U$ test was 
Table 1. Participant characteristics for each organogram condition.

\begin{tabular}{|c|c|c|c|c|c|}
\hline & $\begin{array}{l}\text { Egalitarian organogram } \\
\qquad(n=21)\end{array}$ & $\begin{array}{l}\text { Low rank hierarchical organogram } \\
(n=23)\end{array}$ & $\begin{array}{l}\text { High rank hierarchical organogram } \\
\qquad(n=22)\end{array}$ & $\mathrm{F} / \mathrm{X}^{2} /$ Fischer's & $\bar{p}$ \\
\hline Age $[M(S D)]$ & $35.25(15.90)$ & $37.64(12.30)$ & $36.40(16.08)$ & 0.14 & .87 \\
\hline Gender [n female (\%)] & $12(57.1)$ & $15(65.2)$ & $17(77.3)$ & 1.99 & .37 \\
\hline NART error [M(SD)] & $3.33(2.01)$ & $2.65(1.97)$ & $2.57(2.73)$ & 0.73 & .49 \\
\hline ROCF $[M(S D)]$ & $28.67(2.95)$ & $28.93(4.18)$ & $30.43(2.80)$ & 1.72 & .19 \\
\hline \multicolumn{6}{|l|}{ Educational history [n (\%)] } \\
\hline UK A level's or equivalent & $8(38.1)$ & $3(13.0)$ & $5(22.7)$ & 10.61 & .31 \\
\hline Bachelor's degree & $4(19.0)$ & $10(43.5)$ & $8(36.4)$ & & \\
\hline Master's degree & $6(28.6)$ & $4(17.4)$ & $5(22.7)$ & & \\
\hline PhD & $1(4.8)$ & $1(4.3)$ & $1(4.5)$ & & \\
\hline Other & $0(0)$ & $2(8.7)$ & $1(4.5)$ & & \\
\hline Missing & $2(9.5)$ & $3(13.0)$ & $2(9.1)$ & & \\
\hline \multicolumn{6}{|l|}{ Employment status [n (\%)] } \\
\hline Unemployed & $2(9.5)$ & $4(17.4)$ & $3(13.6)$ & 3.13 & .57 \\
\hline Employed & $9(42.9)$ & $14(60.9)$ & $10(45.5)$ & & \\
\hline Student & $8(38.1)$ & $4(17.4)$ & $7(31.8)$ & & \\
\hline Missing & $2(9.5)$ & $1(4.3)$ & $2(9.1)$ & & \\
\hline \multicolumn{6}{|l|}{ Testing location [n (\%)] } \\
\hline In person & $11(52.4)$ & $11(47.8)$ & $10(45.5)$ & 0.23 & .90 \\
\hline Online & $10(47.6)$ & $12(52.2)$ & $12(54.5)$ & & \\
\hline \multicolumn{6}{|c|}{ Mood/social measures $[M(S D)]$} \\
\hline BDI-II & $4.27(3.58)$ & $4.17(5.10)$ & $3.23(2.86)$ & 0.46 & .63 \\
\hline SCS & $67.65(12.70)$ & $59.74(11.94)$ & $62.22(11.57)$ & 2.45 & .10 \\
\hline SDO & $39.47(12.90)$ & $36.48(13.12)$ & $41.50(10.56)$ & 0.96 & .39 \\
\hline
\end{tabular}

$\mathrm{M}=$ Mean. SD = Standard deviation. NART = National Adult Reading Test. ROCF = Rey-Osterrieth Complex Figure test. BDI-II = Beck Depression Inventory-II. SCS = Social Comparison Scale. SDO = Social Dominance Orientation Scale.

applied. The shapes of the distributions of memory recall scores for the Pooled hierarchy and Egalitarian groups were similar (see supplementary materials).

In support of our first hypothesis, there was a significant difference in memory recall between the Pooled hierarchy and Egalitarian groups (Mann-Whitney $U=312.00, z=$ $-2.26, p=.024$, Cohen's $d=.66$ ) with participants in the Egalitarian condition requiring more attempts to recall the organogram than participants in the Pooled hierarchy condition.

We next examined our second hypothesis that one's place within a hierarchy (HR or LR position) versus one's position in the egalitarian hierarchy would affect memory recall, with recall being better in the HR hierarchical condition. Data were normally distributed for the

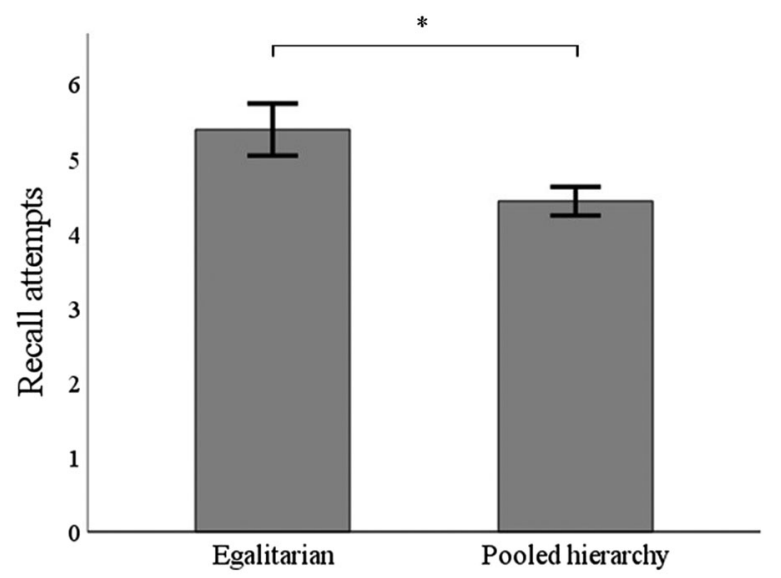

Figure 2. Mean number of recall attempts in the Egalitarian and Pooled hierarchy organogram conditions (LR and HR Hierarchy organograms combined).
Egalitarian and LR hierarchy conditions ( $p s>.05$ ) but not for the HR hierarchy (Shapiro-Wilk W=.91, $p=.04$ ). However, the deviation was within the bounds of what parametric testing is robust to manage (Lix et al., 1996), and so we used ANOVA as intended.

A one-way ANOVA across the three organogram groups (HR hierarchy, LR hierarchy, Egalitarian) revealed a significant main effect of Group on memory recall, $F(2,63)=6.85, \quad p=.002, \quad \eta_{p}^{2}=.18$ (see Figure 3). Paired comparisons with Bonferroni correction (based on the Bonferroni critical value adjustment of the $p$-value) revealed that participants in the HR hierarchy group required significantly fewer recall attempts than those in the Egalitarian $(t(41)=3.61, p=.002$, Cohen's $d=$ $1.13)$, and LR hierarchy conditions ( $t \quad(43)=2.52$, $p=.043$, Cohen's $d=.83$ ). There was no significant difference between the Egalitarian and LR hierarchy conditions $(t(42)=1.16, p=.752$, Cohen's $d=.31)$.

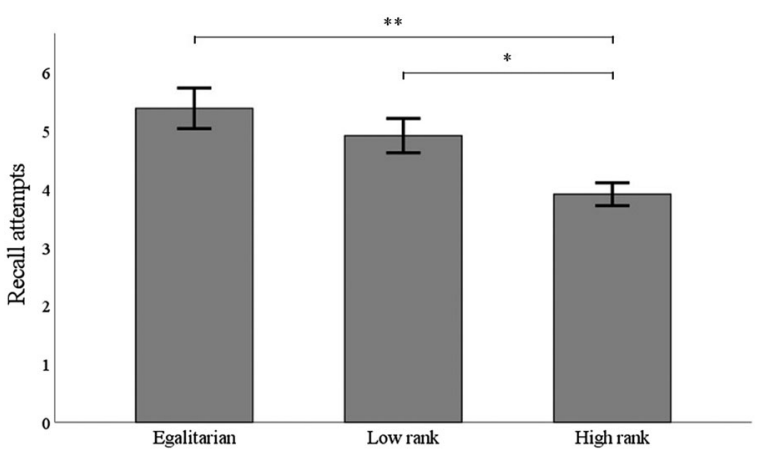

Figure 3. Mean numbers of recall attempts to successfully recall organograms in the Egalitarian, High rank hierarchical and Low rank hierarchical conditions. 


\section{Discussion}

This study provides new evidence describing the ease of processing hierarchical social information. In line with Hypothesis 1, participants memorised organograms faster when they resembled a hierarchy relative to an egalitarian construct. This finding replicates previous work (Zitek \& Tiedens, 2012).

In line with Hypothesis 2, participants with higher positions within the hierarchical organogram learned the organogram structure faster than those in lower positions. We interpret this new finding in terms of self-enhancement bias and personal agency driving task engagement for faster learning. There is compelling evidence that healthy individuals possess an overall positive self-referential bias that extends to self-affirmations (Mezulis et al., 2004), optimism for the future (Sharot, 2011), and selfreferential memories (Anderson et al., 2012; Blaney, 1986; Crary, 1966; Gramzow et al., 2003; Hitchcock et al., 2017; Rubin \& Berntsen, 2003; Silverman, 1964). One account of the present data is that, even in an organogram where the notion of social hierarchy is implicit, being placed in a higher position for many participants will concord with a "rose-tinted" self-serving bias. This could then lead to increased task engagement and cognitive agency resulting in enhanced memory performance (Moore, 2016; Zitek \& Phillips, 2020). Similar to what is reported in the power literature (e.g., Cho \& Keltner, 2020; Keltner et al., 2003; Smith \& Trope, 2006; Trope \& Liberman, 2010), it is the elevated positive affect or enhanced attention towards individuals that are placed in high regard relative to others that may bring a sense of personal agency for influencing attention.

One interesting finding was the reduced and non-significant memory advantage (Cohen's $d=.31$ ) for hierarchical organograms where participants were placed in lowrank positions over egalitarian organograms, relative to the original Zitek and Tiedens (2012) findings and to the present findings involving high rank position. Of course, the Zitek and Tiedens studies did not manipulate the participants' own hierarchical position and it may be that placing participants in a low rank position actually interfered with memory performance as it contradicted putative self-enhancement biases. Further work is needed to elucidate this effect.

Additional future work could investigate whether this effect is eliminated or reversed in individuals who perceive their own social rank as low. For example, adults with persistent depression have been shown to present with behaviours and traits that are characteristic of low rank (Allan \& Gilbert, 1995, 1997; Allen \& Badcock, 2003; Gilbert \& Allan, 1998; Gilbert, 2000; Zuroff et al., 2007). It is possible that individuals with self-perceived low social rank will show better memory performance for social hierarchical information when positioned with a self-congruent low rank within the hierarchy.
One issue worth noting is the transfer of the study to online testing as a result of the COVID-19 pandemic. To verify that this did not affect the results, our analyses were repeated whilst covarying for mode of testing (along with other putative confounds), and the results were the same as for the main analyses and with slightly stronger effect sizes (see Supplementary Materials).

A potential limitation of the study concerns various structural aspects of the organograms that participants learned. For the HR Hierarchy organogram, it is plausible that participants felt that they would be in some kind of interactive relationship with all of the people below them in the hierarchy as might be the case if this was an organisational chart for a workplace. In contrast, participants in the LR Hierarchy condition may have implicitly assumed that they would interact only with the people represented at the next immediately higher level in the organogram. These different implicit assumptions would convey different distributions of importance across the organogram nodes and could have contributed to the differences in learning rates. Similarly, the LR Hierarchy organogram contains fewer associative links from the participant's node to other members of the hierarchy relative to the HR organogram. This was done to preserve the hierarchical appearance of the organograms based on piloting. However, the presence of more associations in the HR Hierarchy organogram could have facilitated learning rates relative to the LR Hierarchy organogram. Follow-on experiments systematically manipulating these variables are now indicated to quantify and elucidate any such effects.

In summary, we replicated previous research (Zitek \& Tiedens, 2012) that memory for hierarchical information is better relative to egalitarian information. Furthermore, we showed that participants presented in a high-rank position within a hierarchy memorised the information for that hierarchy faster than participants placed in low rank positions, suggesting that biases in memory will favour hierarchical information if modulated by perceptions of one's own social rank.

\section{Note}

1. Participants also completed a number of other affect and social questionnaires as part of a larger study. Further information on the other questionnaires are available online.

\section{Acknowledgements}

We would like to thank team members from the MRC Cognition \& Brain Sciences Unit for their comments on an earlier version of this manuscript.

\section{Disclosure statement}

No potential conflict of interest was reported by the author(s). 


\section{Funding}

This work was funded by the The Medical Research Council (Grant Reference: SUAG/043 G101400) and partly supported by the National Institute for Health Research Cambridge Biomedical Research Centre. Medical Research Council (MRC) Cognition \& Brain Sciences Unit (CBU).

\section{Ethics approval and consent to participate}

The study was approved by The Cambridge University Psychology Research Ethics committee. At the beginning of the study, informed consent to participate was obtained from each individual. In addition, each participant was debriefed at the end of the study.

\section{ORCID}

Kirsty Griffiths (D) http://orcid.org/0000-0001-7158-2683

\section{References}

Alicke, M. D. (1985). Global self-evaluation as determined by the desirability and controllability of trait adjectives. Journal of Personality and Social Psychology, 49(6), 1621-1630. https://doi.org/10.1037/ 0022-3514.49.6.1621

Alicke, M. D., Klotz, M. L., Breitenbecher, D. L., Yurak, T. J., \& Vredenburg, D. S. (1995). Personal contact, individuation, and the better-than-average effect. Journal of Personality and Social Psychology, 68(5), 804-825. https://doi.org/10.1037/0022-3514.68. 5.804

Allan, S., \& Gilbert, P. (1995). A social-comparison scale - Psychometric properties and relationship to psychopathology. Personality and Individual Differences, 19(3), 293-299. https://doi.org/10.1016/ 0191-8869(95)00086-L

Allan, S., \& Gilbert, P. (1997). Submissive behaviour and psychopathology. British Journal of Clinical Psychology, 36(4), 467-488. https:// doi.org/10.1111/j.2044-8260.1997.tb01255.x

Allen, N. B., \& Badcock, P. B. T. (2003). The social risk hypothesis of depressed mood: Evolutionary, psychosocial, and neurobiological perspectives. Psychological Bulletin, 129(6), 887-913. https://doi. org/10.1037/0033-2909.129.6.887

Anderson, C., Brion, S., Moore, D. A., \& Kennedy, J. A. (2012). A statusenhancement account of overconfidence. Journal of Personality and Social Psychology, 103(4), 718-735. https://doi.org/10.1037/ a0029395

Anderson, C., Hildreth, J. A. D., \& Howland, L. (2015). Is the desire for status a fundamental human motive? A review of the empirical literature. Psychological Bulletin, 141(3), 574-601. https://doi.org/10. 1037/a0038781

Bellah, R. N., Madsen, R., Sullivan, W. M., Swindler, A. and Tipton, S. M. (1996). Habits of the heart: Individualism and commitment in American life. Updated Edition. University of California Press.

Blaney, P. H. (1986). Affect and memory - a review. Psychological Bulletin, 99(2), 229-246. https://doi.org/10.1037/0033-2909.99.2.229

Brewer, M. B., \& Gardner, W. (1996). Who is this" We"? levels of collective identity and self representations. Journal of Personality and Social Psychology, 71(1), 83-93. https://doi.org/10.1037/00223514.71.1.83

Brown, J. D. (1986). Evaluations of self and others - self-enhancement biases in social judgments. Social Cognition, 4(4), 353-376. https:// doi.org/10.1521/soco.1986.4.4.353

Cho, M., \& Keltner, D. (2020). Power, approach, and inhibition: Empirical advances of a theory. Current Opinion in Psychology, 33, 196-200. https://doi.org/10.1016/j.copsyc.2019.08.013
Crary, W. G. (1966). Reactions to incongruent self-experiences. Journal of Consulting Psychology, 30(3), 246-252. https://doi.org/10.1037/ h0023347

Crivelli, C., \& Fridlund, A. J. (2018). Facial displays Are tools for social influence. Trends in Cognitive Sciences, 22(5), 388-399. https://doi. org/10.1016/j.tics.2018.02.006

Gilbert, P. (2000). The relationship of shame, social anxiety and depression: The role of the evaluation of social rank. Clinical Psychology \& Psychotherapy: An International Journal of Theory \& Practice, 7(3), 174-189. https://doi.org/10.1002/1099-0879 (200007)7:3<174::AID-CPP236>3.0.CO;2-U

Gilbert, P., \& Allan, S. (1998). The role of defeat and entrapment (arrested flight) in depression: An exploration of an evolutionary view. Psychological Medicine, 28(3), 585-598. https://doi.org/10. 1017/s0033291798006710

Gramzow, R. H., Elliot, A. J., Asher, E., \& McGregor, H. A. (2003). Selfevaluation bias and academic performance: Some ways and some reasons why. Journal of Research in Personality, 37(2), $41-$ 61. https://doi.org/10.1016/S0092-6566(02)00535-4

Hitchcock, C., Rees, C., \& Dalgleish, T. (2017). The devil's in the detail: Accessibility of specific Personal memories supports rose-tinted self-generalizations in mental health and toxic self-generalizations in clinical depression. Journal of Experimental Psychology-General, 146(9), 1286-1295. https://doi.org/10.1037/xge0000343

Keltner, D., Gruenfeld, D. H., \& Anderson, C. (2003). Power, approach, and inhibition. Psychological Review, 110(2), 265-284. https://doi. org/10.1037/0033-295x.110.2.265

Lewinsohn, P. M., Mischel, W., Chaplin, W., \& Barton, R. (1980). Social competence and depression - the role of illusory self-perceptions. Journal of Abnormal Psychology, 89(2), 203-212. https://doi.org/10. 1037/0021-843x.89.2.203

Lix, L. M., Keselman, J. C., \& Keselman, H. J. (1996). Consequences of assumption violations revisited: A quantitative review of alternatives to the one-way analysis of variance $F$ test. Review of Educational Research, 66(4), 579-619. https://doi.org/10.3102/ 00346543066004579

Magee, J. C., \& Galinsky, A. D. (2008). Social hierarchy: The self-reinforcing nature of power and status. Academy of Management Annals, 2(1), 351-398. https://doi.org/10.1080/19416520802211628

Mezulis, A. H., Abramson, L. Y., Hyde, J. S., \& Hankin, B. L. (2004). Is there a universal positivity bias in attributions? A meta-analytic review of individual, developmental, and cultural differences in the self-serving attributional bias. Psychological Bulletin, 130(5), 711-747. https://doi.org/10.1037/0033-2909.130.5.711

Moore, J. W. (2016). What is the sense of agency and why does it matter? Frontiers in Psychology, 7, 1272. https://doi.org/10.3389/ fpsyg.2016.01272

Nelson, H. E., \& Willison, J. (1991). National adult reading test (NART). Nfer-Nelson.

Osterrieth, P. A. (1944). Le Test du copie d'une figure complexe/ Test of coping a complex figure; Contribution to the study of perception and memory. Archives of Psychology, 30, 206-356.

Rubin, D. C., \& Berntsen, D. (2003). Life scripts help to maintain autobiographical memories of highly positive, but not highly negative, events. Memory \& Cognition, 31(1), 1-14. https://doi.org/10.3758/ Bf03196077

Sharot, T. (2011). The optimism bias. Current Biology, 21(23), R941R945. https://doi.org/10.1016/j.cub.2011.10.030

Silverman, I. (1964). Self-Esteem and differential responsiveness to success and failure. Journal of Abnormal and Social Psychology, 69(1), 115-119. https://doi.org/10.1037/h0039858

Smith, P. K., \& Trope, Y. (2006). You focus on the forest when you're in charge of the trees: Power priming and abstract information processing. Journal of Personality and Social Psychology, 90(4), 578596. https://doi.org/10.1037/0022-3514.90.4.578

Tarr, E. K., \& Kohles, J. C. (2019). Why Pay attention to You? The role of moderation effects on the power-attention relationship. Journal of 
Organizational Psychology, 19(2), 144-160. https://doi.org/10. 33423/jop.v19i2.2050

Taylor, S. E., \& Brown, J. D. (1988). Illusion and well-being - a social psychological perspective on mental-health. Psychological Bulletin, 103(2), 193-210. https://doi.org/10.1037/0033-2909.103.2.193

Trope, Y., \& Liberman, N. (2010). Construal-level theory of psychological distance. Psychological Review, 117(2), 440-463. https://doi.org/ 10.1037/a0018963

Zink, C. F., Tong, Y. X., Chen, Q., Bassett, D. S., Stein, J. L., \& MeyerLindenberg, A. (2008). Know your place: Neural processing of social hierarchy in humans. Neuron, 58(2), 273-283. https://doi. org/10.1016/j.neuron.2008.01.025

Zitek, E. M., \& Phillips, L. T. (2020). Ease and control: The cognitive benefits of hierarchy. Current Opinion in Psychology, 33, 131-135. https://doi.org/10.1016/j.copsyc.2019.07.015

Zitek, E. M., \& Tiedens, L. Z. (2012). The fluency of social hierarchy: The ease with which hierarchical relationships are seen, remembered, learned, and liked. Journal of Personality and Social Psychology, 102(1), 98-115. https://doi.org/10.1037/ a0025345 